\title{
Charles coutel (éd.), Le fanatisme religieux du XVI siècle à nos jours. Étudier, comprendre, prévenir
} Avec la collaboration de Mokhtar Ben Barka et Olivier Rota, Arras, Artois Presses Université, 2017, 181 p.

\section{Stéphanie Tremblay}

\section{OpenEdition}

1 Journals

\section{Édition électronique}

URL : https://journals.openedition.org/assr/44738

DOI : $10.4000 /$ assr.44738

ISSN : $1777-5825$

Éditeur

Éditions de l'EHESS

\section{Édition imprimée}

Date de publication : 1 décembre 2018

Pagination : 245-247

ISSN : 0335-5985

\section{Référence électronique}

Stéphanie Tremblay, « Charles coutel (éd.), Le fanatisme religieux du xvi e siècle à nos jours. Étudier, comprendre, prévenir », Archives de sciences sociales des religions [En ligne], 184 | octobre-décembre 2018, mis en ligne le 01 décembre 2018, consulté le 12 janvier 2022. URL : http:// journals.openedition.org/assr/44738; DOI : https://doi.org/10.4000/assr.44738 


\section{Charles couTEL (éd.), Le fanatisme religieux du XVI ${ }^{\mathrm{e}}$ siècle à nos jours. Étudier, comprendre, prévenir}

Avec la collaboration de Mokhtar Ben Barka et Olivier Rota, Arras, Artois Presses Université, 2017, 181 p.

Stéphanie Tremblay

\section{RÉFÉRENCE}

Charles CoUTEL (éd.), Le fanatisme religieux du XVI ${ }^{\mathrm{e}}$ siècle à nos jours. Étudier, comprendre, prévenir. Avec la collaboration de Mokhtar Ben Barka et Olivier Rota, Arras, Artois Presses Université, 2017, 181 p.

1 Pourquoi parler de "fanatisme" dans ce récent livre alors que la notion de « radicalisation » est sur toutes les lèvres? Y aborde-t-on des enjeux analogues à ceux que d'autres auteurs analysent à partir du concept de radicalisation ou bien y traite-ton d'une problématique différente? Les auteurs dont les études sont ici réunies par Charles Coutel, à la suite d'un colloque tenu le 27 février 2015, proposent d'étudier le fanatisme en vue, notamment, de mieux comprendre « les processus de radicalisation de certains jeunes, après les attentats de 2015 et 2016 »(p.13), dont les premiers à Charlie Hebdo, sont survenus tout juste après le colloque. Mais, peut-être par souci d'éviter d'aborder frontalement cette question brûlante ayant éprouvé de trop près la population française, les études proposées puisent surtout au passé des $\mathrm{XVI}^{\mathrm{e}}$, $\mathrm{XVII}^{\mathrm{e}}$ et $\mathrm{XVIII}^{\mathrm{e}}$ siècles et privilégient la catégorie analytique du fanatisme religieux. Cette notion, connue, mais moins utilisée dans la recherche récente, présente ici l'avantage de se démarquer d'un buzzword politiquement chargé tout en apportant un éclairage nouveau et pertinent à des questions restées actuelles. Le fanatisme est ici pensé et conceptualisé dans la matrice chrétienne, tout particulièrement dans ses déclinaisons protestantes aux XVI ${ }^{e}$ et $\mathrm{XVII}^{\mathrm{e}}$ siècles, puis dans son rapport au catholicisme, aux XVII ${ }^{\mathrm{e}}$ et 
$\mathrm{XVIII}^{\mathrm{e}}$ siècles. Aux relations entre les fanatiques et leurs dénonciateurs se superposent ainsi les rapports de pouvoir entre dominants et dominés, État, clergé et mouvements contestataires. Le fanatisme dont il est question ici trace donc des frontières poreuses entre religion et politique, élites bien-pensantes et minorités subversives.

2 Les contributions rassemblées visent à aborder conjointement trois questions centrales : comment étudier, comprendre et prévenir le fanatisme. D'abord, à quoi renvoie précisément le fanatisme? Une première définition de travail, issue de Paul Zawadzki (2010), associe cette notion à sa dimension polémique ainsi qu'à sa prétention à vouloir confondre ce qui est avec ce qui doit être: «Forgé par l'anti-fanatisme, il fut introduit dans la modernité par la critique libérale puis popularisé par les Lumières [...]. Le fanatisme manifeste l'impatience de rabattre le ciel sur la terre en télescopant l'universel et le particulier» (p. 13). Pour l'étudier, une hypothèse, servant aussi de prémisse, traverse l'ouvrage: "Le fanatisme s'est constitué en objet critique de recherche tout au long du siècle des Lumières " (p. 12). À travers trois moments de la philosophie des Lumières, marqués respectivement par l'Encyclopédie, Voltaire puis Kant et Condorcet, Coutel soutient que le fanatisme s'est constitué comme objet de réflexion et d'analyse, orienté autour de ses conditions d'émergence, et non plus seulement invectivé, craint ou considéré dans ses conséquences délétères. Au fil de ces trois moments, le fanatisme passe donc d'une conception critique, associant cette attitude de zèle (religieux) à un esprit superstitieux et ignorant, à une conception autocritique, "faisant de la superstition une menace permanente guettant tout esprit, fût-il rationnel ou philosophique» (p. 16). Avec Kant et Condorcet, la mise en garde voltairienne est enchâssée dans un projet philosophique et éducatif orienté vers «la coexistence rationnelle et universelle des libertés individuelles éclairées » (p.17) et tourné vers l'avenir.

3 La compréhension du fanatisme passe ensuite par l'analyse chronologique de divers cas de figures historiques du protestantisme qui illustrent à la fois sa labilité dans le temps et l'espace et la rémanence des mêmes marqueurs cognitifs et sociaux d'inconditionnalité et de polarisation. Les quatre premiers textes se concentrent sur des figures protestantes. Samuel Tomeï s'intéresse à Sébastien Castellion (1515-1563) qui, en pointant les limites de l'hypothèse à l'origine du livre, fait preuve avant les Lumières d'une pensée libre et critique à l'égard de l'interprétation calviniste de la Bible, érigée comme la norme en son temps. Il remet en effet vivement en question ce que Ferdinand Buisson appellera plus tard «la folie du sens propre» (p.30) en privilégiant une lecture morale et spirituelle de la Bible plutôt que littérale et en critiquant le dogme de la prédestination au profit de la liberté et de la responsabilité humaines. Puis, Alain Jobin trace le portrait de femmes protestantes considérées comme "fanatiques» (Jeanne d'Albret, Madeleine de Mailly, etc.), qui ont dans plusieurs cas poussé à l'extrême leur foi en même temps que leur colère et se sont dressées haut et fort contre toutes sortes de persécutions politiques ou religieuses, certaines au prix d'une violence retournée vers l'adversaire. Le texte de Chady Hage-Ali nous transporte en Nouvelle-Angleterre aux premiers temps de la colonie des puritains calvinistes cherchant à établir la "Nouvelle Jérusalem ». L'auteur illustre bien le passage parfois observé entre persécuté et persécuteur, dominé et dominant et les points aveugles que ces aller-retour historiques peuvent comporter, en montrant comment la cristallisation politique des conceptions calvinistes a fini par évacuer tout autre groupe, jugé dissident et fanatique. L'épisode terrifiant des sorcières de Salem, 
catalysé par la crise sociale et existentielle polarisant la ville, continue de montrer que le fanatisme mute et prend souvent comme bouc émissaire le minoritaire, le plus faible, en liguant la majorité derrière des arguments parfois imprévisibles, tel que la vision des spectres (spectral evidence). L'étude suivante, de Cyril Selzner, aborde le cas paradoxal des Quakers au XVII e siècle, qui, taxés de "fanatiques" par les pouvoirs officiels, sont certes porteurs d'une contestation radicale du clergé jugé corrompu, mais ne passent pas à l'acte violent. Leur " fanatisme pacifique » a d'ailleurs fait l'objet d'une réflexion bienveillante de Voltaire. Cette contribution pose, à notre sens, une question cruciale dans les débats contemporains sur les signes religieux ostentatoires en abordant la nature de la violence symbolique des Quakers, qui gardent en tout temps leur chapeau sur leur tête ou refusent de se plier aux rituels de politesse : "On aurait tort de sousestimer cette violence-là, qui est loin d'être purement symbolique pour leurs contemporains : le refus de jouer le jeu social [...] c'est également un vivant et perpétuel reproche à tous ceux qui ne suivent pas le droit chemin, celui de la "Vérité" " (p. 85). L'intervention suivante éprouve les limites de la compréhension en examinant le cas du curé Jean-Baptiste L'Ouvreleul, auteur de L'histoire du fanatisme renouvelé, dont la critique de la révolte "fanatique» des protestants est imperméable à toute compréhension, étant elle-même aveuglée par la sidération, par le rejet de la « folie » et de la "possession diabolique» des protestants. Avec Bruno Béthouart qui aborde la déchristianisation en Boulonnais, on s'intéresse en modernité au "transfert de sacralité » du champ religieux vers le champ politique en s'interrogeant en filigrane sur les apories de ce nouveau fanatisme issu d'un âpre combat pour la liberté. L'auteur conclut en suggérant qu'une tradition d'irréligion subsiste dans certains segments de la culture dominante, ce qui peut encore nous laisser méditer aujourd'hui sur certaines contradictions de la laïcité. Ce tableau historique met bien en scène la tendance à taxer de fanatique le plus faible, le minoritaire, l'illégitime, en particulier en contexte de crise. Mais, il laisse aussi voir, par rétroanalyse, que le fanatisme est souvent mobile en circulant d'un groupe à l'autre, y compris en créant de nouvelles frontières.

Pour prévenir le fanatisme, Jean-Luc Blaquart relit le philosophe Alain (Émile Chartier), pour qui ce «mal humain» ne peut être jugulé qu'en se dégageant d'une pensée sans nuance ou manichéiste. Pour lui, le fanatisme peut être déjoué par le rire et l'art, qui délivrent des registres du bien ou du vrai : « Le rire souligne les limites du savoir ou de la volonté, ce qui explique pourquoi il n'est pas supportable par le fanatique » (p. 118). Dans sa conclusion générale, Coutel prolonge cette intuition humaniste en proposant une consolidation de l'idéal fraternel des Lumières. Pour lui, il ne suffit pas d'énoncer la fraternité dans l'idéal démocratique, mais bien de la vivre. Aussi, puisant à l'analyse de Gauchet, il se méfie du « retour subreptice de l'hétéronomie dans l'autonomie et de la transcendance dans l'immanence dans la définition et la pratique de la fraternité » (p.135). Pour se prémunir contre ce retour virtuel du paternalisme qui fragiliserait le "vivre-ensemble", Coutel plaide pour une éthique de la sollicitude: "Appelons sollicitude la vertu relationnelle éthique, non invasive, requise par ce dispositif de l'hospitalité. La sollicitude est tournée vers l'avenir, n'envahit pas l'autre et ne cherche pas à "se mettre à sa place" ». Ainsi, selon Coutel, « l'hospitalité, remplie de sollicitude, devient la condition a priori et nécessaire de la fraternité consciente de soi et peut contribuer à faire reculer le fanatisme religieux, voire à le prévenir » (p. 140).

5 Si la triple tâche énoncée au départ est amplement remplie par cet ouvrage dont l'abondante documentation historique, la rigueur des études réunies et l'originalité des regards posés sur l'objet d'étude sont manifestes, une question cruciale ne nous semble 
que partiellement élucidée : celle de la rationalité du fanatique. Les textes suggèrent en effet de diverses manières que n'importe quel individu raisonnable peut "basculer " dans cette attitude de zèle, se laissant alors happer par " une pensée sans aucun doute " (p. 123), comme s'il s'agissait là d'un aller sans retour. Or, le fanatique est-il vraiment dépourvu de rationalité, entendue comme la capacité de s'autocritiquer? Quelques travaux sur la dynamique des croyances (Gerald Bronner, Romy Sauvayre) concluent au fait que même chez des personnes ayant adhéré de manière inconditionnelle à un système de croyances dit "extrême ", la rationalité n'est pas totalement éclipsée. En cas de conflit axiologique avec ses valeurs centrales, le doute peut d'ailleurs fissurer brusquement des croyances "extrêmes » et même les faire voler en éclats. De même, les raisons de croire ne sont pas nécessairement mortifères. Ainsi en va-t-il de certaines croyances descriptives sur la destinée ou des convictions religieuses tournées vers une communauté refuge (par exemple, juifs hassidim). La radicalisation peut aussi être cognitive ou comportementale, sans qu'il y ait de continuité entre les deux. On peut regretter que la catégorie de fanatisme n'introduise pas les nuances nécessaires entre ces deux formes d'extrémisme. De cette limite conceptuelle découle une question éthique: dans un contexte démocratique protégeant la liberté de conscience de la tyrannie de la majorité, peut-on vraiment intervenir sur les formes cognitives d'extrémisme sans franchir l'ultime garde-fou de l'intimité individuelle? 